\title{
Effects of Temperature Gradients, Self-Absorption, and Line Shape on Apparent Rotational Temperatures of $\mathrm{OH}^{*}$
}

\author{
B. H. Elliotet and S. S, PeNner \\ Guggenheim Jet Propulsion Center, California Insitute of Technology, Pasadena, California
}

(Received April 3, 1953)

\begin{abstract}
The effect on apparent rotational temperatures (of $\mathrm{OH}$ ) of adjacent radiating and absorbing regions at different temperatures and of spectral line shape, coupled with varying degrees of self-absorption, has been studied. The calculations emphasize the fact that definitive conclusions regarding interpretation of flame spectra are difficult to obtain by use of conventional low-resolution spectroscopic studies of flames. Multiple path experiments, or absorption studies with a discrete line source, appear promising provided they are restricted to conditions under which the spect ral line shape is known.
\end{abstract}

$I^{\top}$ $N$ earlier publications the influence of spectral line shape $^{l}$ and of self-absorption ${ }^{1,2}$ on apparent rotational temperatures of $\mathrm{OH}$ has been studied. It is the purpose of the present calculations to amplify the conclusions drawn previously, particularly as regards distortion produced by adjacent radiating and absorbing regions at different temperatures.

\section{EFFECT OF LINE SHAPE AND OF SELF-ABSORPTION ON THE USE OF THE ISOINTENSITY METHOD ${ }^{3}$ FOR ISOTHERMAL SYSTEMS}

According to the isointensity method the temperature of a radiator is obtained from a comparison of two spectral lines which are of equal intensity. Let $A(a, K)$ be the total intensity of the line identified by the index $K$. The line shape for combined Doppler and collision broadening is described by the parameter

$$
a=\left(b_{N}+b_{C}\right)(\ln 2)^{\frac{1}{2}} / b_{D},
$$

where $b_{N}, b_{C}$, and $b_{D}$ denote, respectively, the natural, the collision, and Doppler half-widths. In general

\footnotetext{
* Supported by the U. S. Office of Naval Research under Contract Nonr-220(03), NR $015-210$.

+ This article is based, in part, on a thesis submitted by Lt. Colonel Elliott to the Graduate School of the California Institute of Technology, in partial fulfillment of requirements for the degree of Aeronautical Engineer, June, 1953.

is. S. Penner, J. Chem. Phys. 21, 686 (1953).

${ }^{2} \mathrm{~S}$. S. Penner, J. Chem. Phys 21, 31 (1953).

${ }^{3}$ G. H. Dieke and H. M. Crosswhite, The Ultrantolet Bands of OH, Fundamental Dati (Bumblebee Series Report No. 87, November, 1948).
}

$b_{N} \ll b_{C}$ and $a \simeq 0$ for pure Doppler broadening. The effect of line shape, under isothermal conditions, on the use of the isointensity method may be determined by evaluating the ratio $A(a, K) / A\left(a, K^{\prime}\right){ }^{4}$ The result can be obtained most conveniently by using the "curves of growth.".s

The results for $K^{\prime}=1$ of representative calculations at $3000^{\circ} \mathrm{K}$ for the $P_{1}$-branch, $(0,0)$ band, ${ }^{2} \longrightarrow \rightarrow^{2} \Pi$ transitions of $\mathrm{OH}$ are plotted in Figs. 1 and 2 for $a=0.005$, and $a=2$, respectively. The self-absorption parameter $\epsilon^{\prime}$ refers to the value of $1-\exp \left(-P_{\max } X\right)$ for the first line of the $P_{1}$ branch. ${ }^{4}$ In order to illustrate the combined effects of line shape and self-absorption on lines of equal intensity, from which conclusions might be drawn concerning the "temperature," the results listed in Table I may be consulted. In Table I the lines of intensity closest to the intensities of the lines with $K=3$ and $K=6$ have been tabulated for different values of $\epsilon^{\prime}$ and of $a$. Reference to the data shown in Table I indicates a large effect of $\epsilon^{\prime}$ for small $a$, but the results are quite insensitive to $\epsilon^{\prime}$ for large $a$. Hence the conclusion is reached that self-absorption errors become more im-

\footnotetext{
${ }^{4}$ For discussions of terms which are not considered in detail in the present publication, references 1 and 2 should be consulterl. ${ }^{5}$ E. M. F. van der Held, Z. Physik 70, 508 (1931); A Unsöld, Physik der Stematmosphären (J. W. Edwards Company, Ann Arbor, Michigan, 1948), p. 168; for an extension of the curves of growth to larger values of the line-shape parameter $a$, see S. S Penner and R. W. Kavanagh, Technical Report No. 6, Contract Nonr-220(03), NR 015 210, California Institute of Technology, Pasadena (September, 1952).
} 


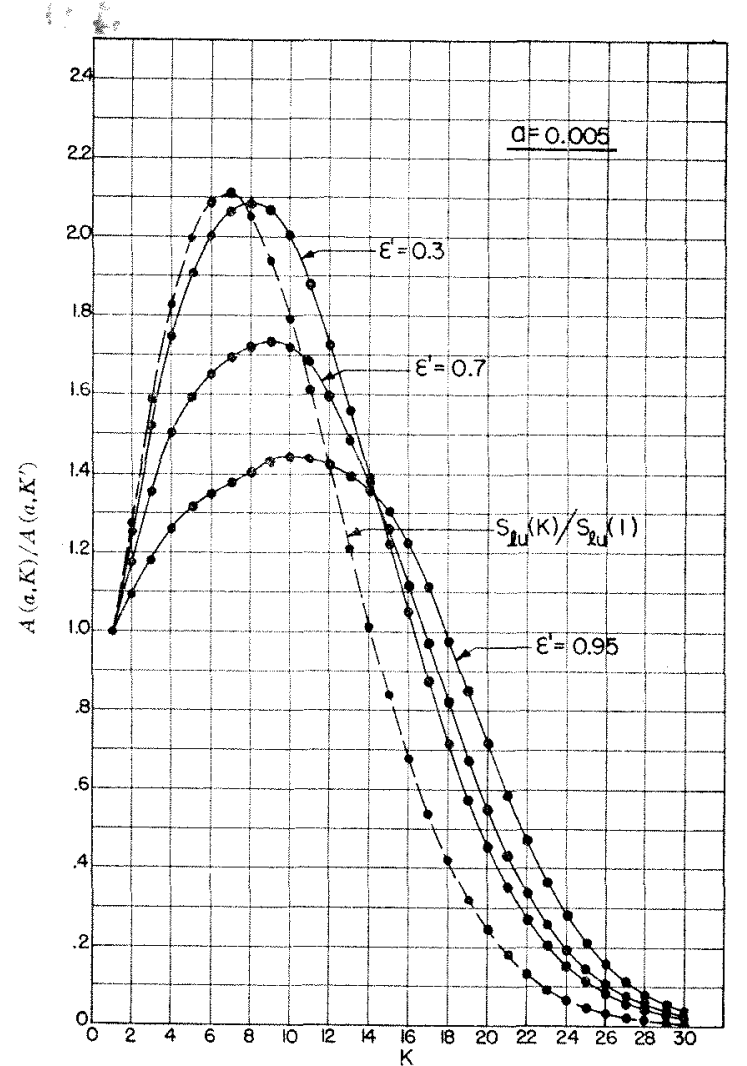

FIG. 1. The quantity $A(a, K) / A\left(a, K^{\prime}\right)$ as a function of $K$ for $K^{\prime}=1, a=0.005$.

portant as the pressure is reduced, i.e., as $a$ is decreased. ${ }^{1}$ A more definite conclusion is justified only if absolute values are known for $a$ in flames, which is not the case at the present time.

\section{TWO-PATH EXPERIMENTS FOR TWO ADJACENT ISOTHERMAL REGIONS (SPECTRAL LINES WITH DOPPLER CONTOUR, PEAK INTENSITIES)}

Calculations which are made in this section correspond to the assumed experimental arrangement illustrated in Fig. 3. The $\mathrm{OH}$ concentration is treated as a variable parameter both in the hot region $\left[\epsilon=1-\exp \left(-P_{\max } X\right)\right]$ and in the cool region $\left[\epsilon^{\prime}=1\right.$ $\left.-\exp \left(-P_{\max } X^{\prime}\right)\right]$. It is physically reasonable to assume $\epsilon^{\prime} \ll \epsilon$. Calculations have been carried out for peak

TABLE I. Effect of $\epsilon^{\prime}$ and $a$ on lines of equal total intensity.

\begin{tabular}{|c|c|c|c|}
\hline & \multicolumn{3}{|c|}{$K$ value of line with intensity closest to $K=3$ for } \\
\hline & $\epsilon^{\prime}=0.3$ & $e^{\prime}=0.7$ & $\epsilon^{\prime}=0.95$ \\
\hline \multirow{5}{*}{$\begin{array}{l}a=0.005 \\
a=0.05 \\
a=2\end{array}$} & 13 & 14 & 16 \\
\hline & 13 & 14 & 16 \\
\hline & 13 & 13 & 14 \\
\hline & \multicolumn{3}{|c|}{$K$ value of line with intensity closest to $K=6$ for } \\
\hline & $\epsilon^{\prime}=0.3$ & $\epsilon^{\prime}=0.7$ & $\epsilon^{\prime}=0.95$ \\
\hline$a=0.005$ & 10 & 11 & 14 \\
\hline$a=0.05$ & 10 & 11 & 13 \\
\hline$a=2$ & 10 & 10 & 10 \\
\hline
\end{tabular}

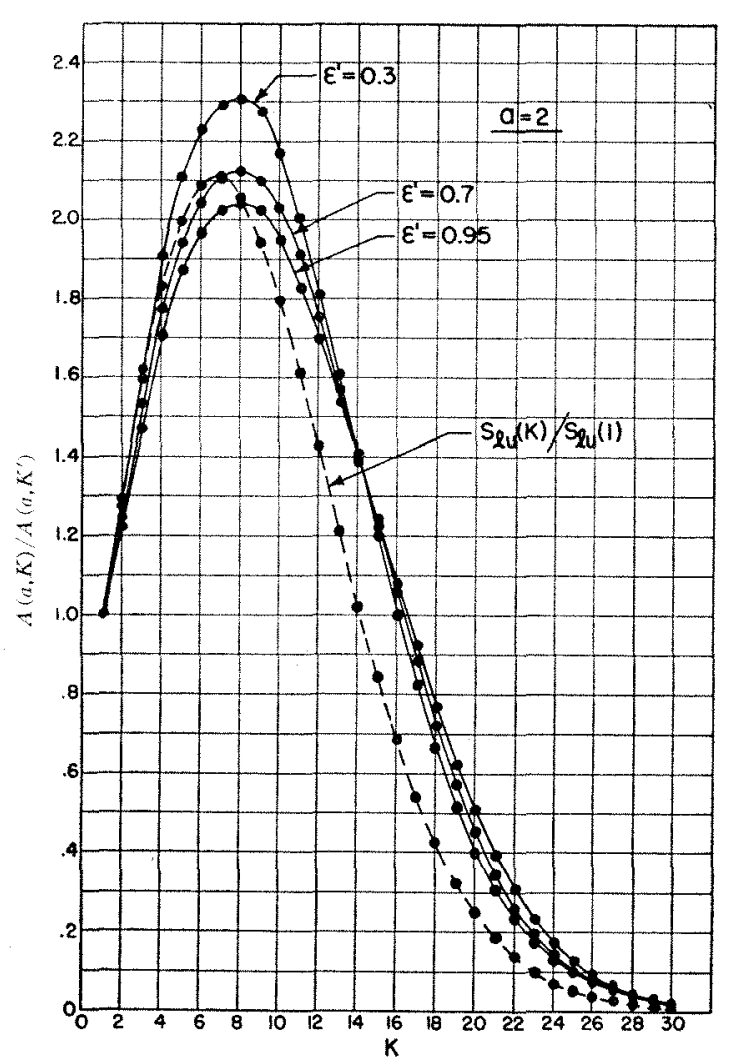

Fig. 2. The quantity $A(a, K) / A\left(a, K^{\prime}\right)$ as a function of $K$ for $K^{\prime}=1, a=2$.

intensities and for spectral lines with Doppler contour. Peak intensities, even for low-pressure flames with Doppler-broadened lines, have not been measured; such measurements might be possible with an interferometer.

Referring to Fig. 3, the peak intensity $I$ for the spectral line whose center lies at $\nu_{l u}$ may be written as

$$
I \simeq R\left(\nu_{l u}\right) \epsilon\left(1-\epsilon^{\prime}\right)\left[1+(1-\epsilon)\left(1-\epsilon^{\prime}\right)^{2}\right]
$$

for unit reflectivity of the mirror, if $\exp \left(-h \nu_{l u} / k T^{\prime}\right)$ $\ll \exp \left(-h \nu_{l u} / k T\right)$. If $I^{\prime}$ represents the intensity for the

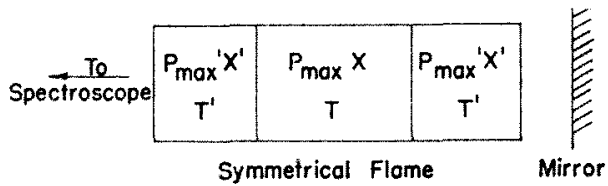

F1G. 3. Schematic arrangement of two-path experiment for a flame represented by two isothermal regions.

single path, then $I^{\prime} \simeq R\left(\nu_{l u}\right) \epsilon\left(1-\epsilon^{\prime}\right)$ whence

$$
I / I^{\prime} \simeq 1+(1-\epsilon)\left(1-\epsilon^{\prime}\right)^{2}
$$

The observable ratio $I / I^{\prime}$ has been calculated as a function of $K$ for various values of $\epsilon(K=1)$ and $\epsilon^{\prime}(K=1)$ for the $P_{1}$ branch, $(0,0)$ band, and ${ }^{2} \Sigma \rightarrow^{2} \Pi$ transitions of OH. Representative results are plotted in Figs. 4 and 5. Reference to these figures shows that the intensity ratio $I(K) / I^{\prime}(K)$ is a sensitive function of the self absorption 


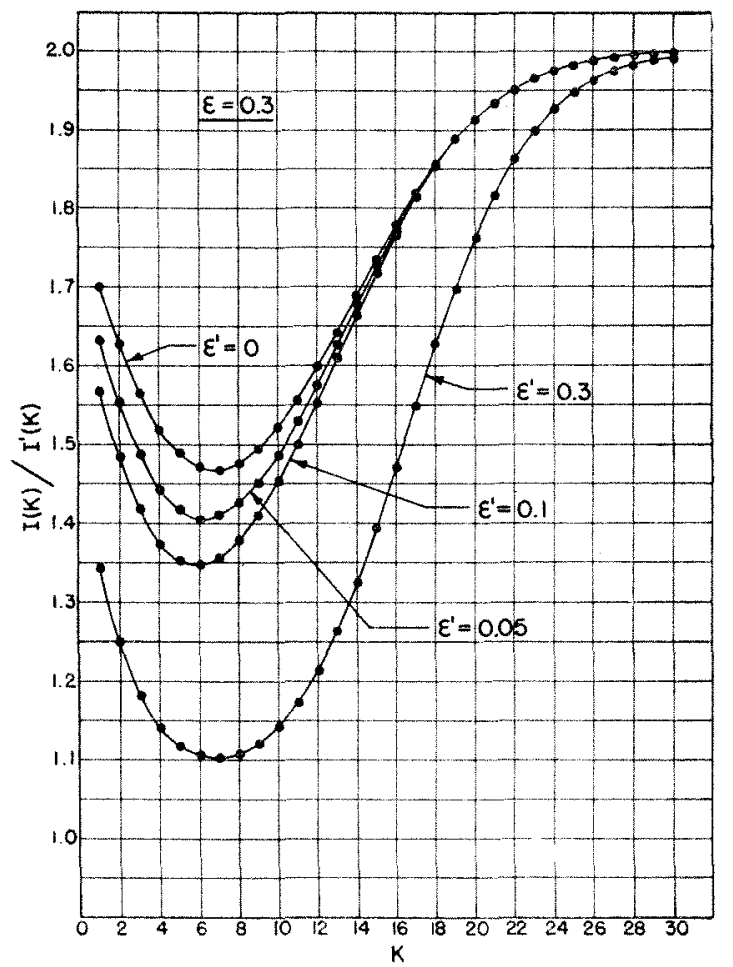

Fig. 4. The quantity $I(K) / I^{\prime}(K)$ as a function of $K$ for $\epsilon=0.3$, $\epsilon^{\prime}=0,0.05,0.10$, and 0.3 .

parameters $\epsilon$ and $\epsilon^{\prime}$. It is clear that the intensity ratio for two-path experiments will be a somewhat less sensitive function of $\epsilon$ and $\epsilon^{\prime}$ for measurements of total intensity.

The important result derived from the present calculations is that even for values of $\epsilon$ as small as 0.3 , the ratio $I(K) / I\left(K^{\prime}\right)$ is appreciably less than two for the stronger lines. In other words, a two-path experiment measuring peak intensities is a relatively sensitive device for studying self-absorption and temperature distortion, quantilatively. As will be shown in Sec. III, this last conclusion applies also to absorption experiments in which peak intensities are measured.

\section{ABSORPTION EXPEWWVWT FOR TWO ADJACEN}

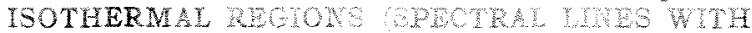
DOPPLER COMOUS, PEAE INESWTHES

Because of the experimental diffculties arising from inadequate resolving power in the measurement of peak intensities for two path experiments, it is of interest to consider the use of absorption experiments using discrete line sources. Calculations which are made in this section correspond to the assumed experimental arrangement illustrated in Fig. 6. The light source is assumed to radiate at the center of a given spectral line as a blackbody at the temperature $T_{s}$, i.e., with intensity $R\left(T_{s}\right)$. For the same conditions for which Eq. (2) applies it is found that

$$
I^{*} / R\left(T_{s}\right) \simeq\left(1-\epsilon^{\prime}\right)^{2}(1-\epsilon),
$$

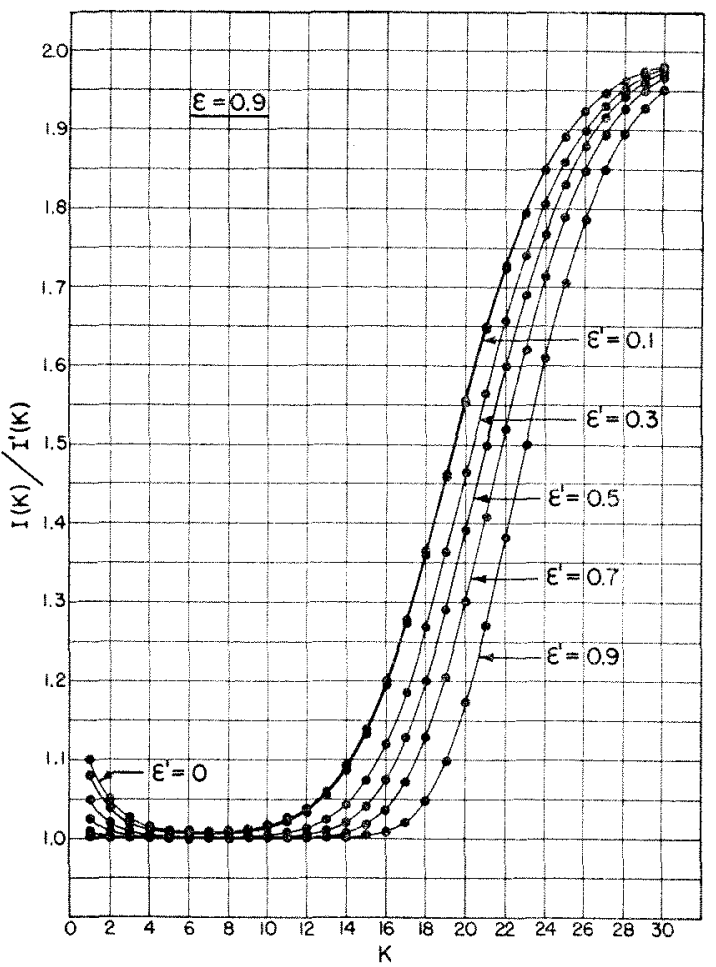

Fro. 5. The quantity $I(K) / I^{\prime}(K)$ as a function of $K$ for $\epsilon=0.9$, $\epsilon^{\prime}=0,0.1,0.3,0.5,0 . \bar{t}$, and 0.9 .

where $I^{*}$ denotes the transmitted intensity at $\nu_{\imath u}$.

Comparison of Eqs. (3) and (4) shows that the transmitted intensity divided by the incident intensity from a discrete line source is nearly equal to $I / I^{\prime}-1$ where $I / I^{\prime}$ is the ratio of the intensity of the flame for a double-path experiment to the intensity of the flame for a single-path experiment. Figures 4 and 5 may then be reinterpreted in such a way that $I / I^{\prime}-1$ represents the transmissivity of the flame for discrete radiation.

Since $I / I^{\prime}$ or $I / I^{\prime}-1$, for measurements of peak intensities, are very sensitive functions of the self-

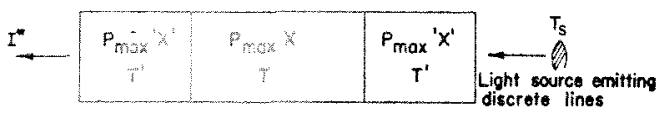

Fo. 6. Schmanc amomocome of absorption experiment for a Tame nemed by wo isothermal regions.

absorption parameters $\varepsilon$ and $\epsilon^{\prime}$, it follows that $I^{*} / R\left(T_{s}\right)$ is also a censitive function of $\epsilon$ and $\epsilon^{\prime}$. Unlike peakintensity determinations in two-path experiments, the discrete line source experiment may be feasible with ordinary spectroscopic apparatus by using as source $\mathrm{OH}$ in a discharge tube, or else an excited metal line which coincides exactly with a line of $\mathrm{OH} \cdot{ }^{8}$ In this connection it is of interest to note that the $3063.97 \mathrm{~A}$ line of tungsten

${ }^{5}$ Absorption experiments using discrete lines as source have been considered at various times by most of the active workers in combustion spectroscopy. See also Kane and Broida, J. Chem. Phys. 21, 347 (1953). 


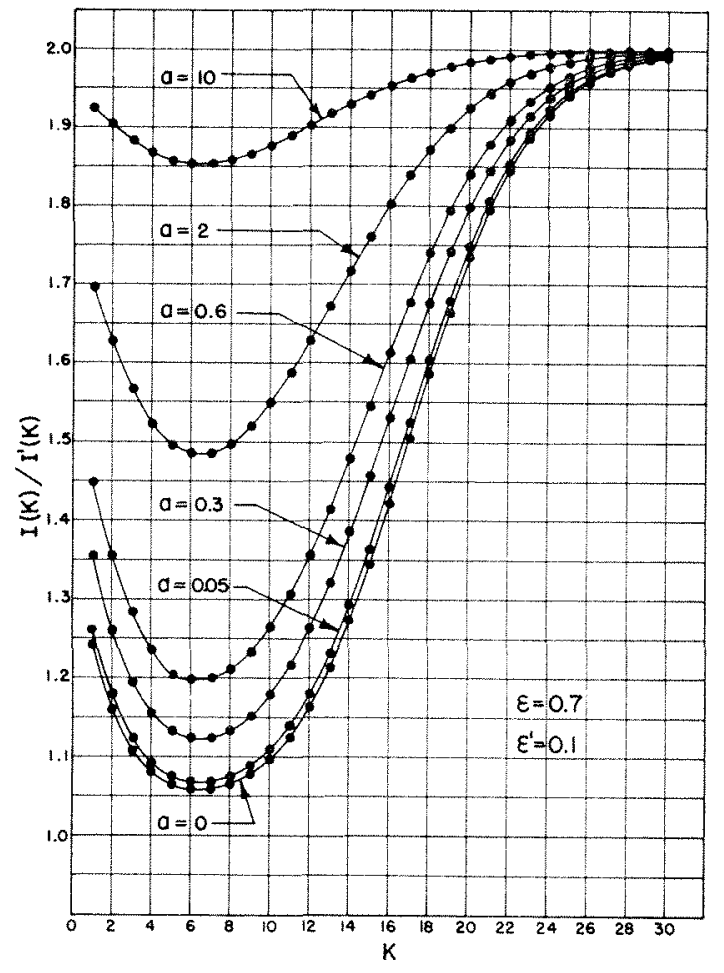

Fic. 7. The quantity $I(K) / I^{\prime}(K)$ as a function of $K$ for $\epsilon=0.7$, $\epsilon^{\prime}=0.1, a=0,0.05,0.3,0.6,2$, and 10 .

coincides with the $10^{\prime}$ line of the $(0,0)$ band, ${ }^{2} \Sigma \rightarrow{ }^{2} I I$ transitions of $\mathrm{OH}$.

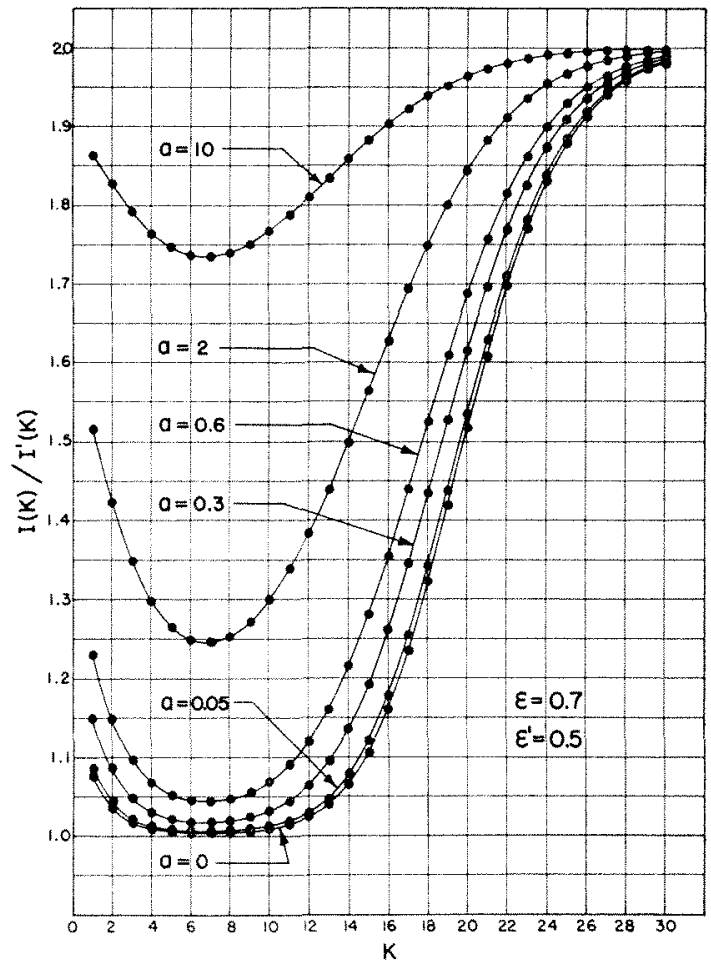

Fic. 8. The quantity $I(K) / I^{\prime}(K)$ as a function of $K$ for $\epsilon=0.7$, $\epsilon^{\prime}=0.5, a=0,0.05,0.3,0.6,2$, and 10 .

\section{TWO-PATH EXPERIMENTS FOR TWO ADJACENT ISOTHERMAL REGIONS (SPECTRAL LINES WITH COMBINED DOPPLER- AND COLLISION- BROADENING, PEAK INTENSITIES)}

The calculations summarized in this section correspond to the experimental arrangement illustrated in Fig. 3. The parameter $P_{\max }$ must now be replaced by $P\left(\nu_{l u}\right)$ where $P\left(\nu_{l u}\right)$, the spectral absorption coefficient at the line center, is to be evaluated for combined Doppler- and collision-broadening. The quantity $P\left(\nu_{l n}\right)$ is related to $P_{\max }$ and $a$ through the expression ${ }^{5}$

where

$$
P\left(\nu_{l u}\right)=P_{\mathrm{m} \mid \mathrm{x} x}\left[\exp \left(a^{2}\right)\right][\operatorname{erfc}(a)]
$$

$$
\operatorname{erfc}(a)=\left[2 / \pi^{(1 / 2)}\right] \int_{a}^{\infty}\left[\exp \left(-x^{2}\right)\right] d x
$$

Equation (3) may be rewritten as

$$
I / I^{\prime} \simeq 1+\left(1-\epsilon_{\mathrm{D}-\mathrm{C}}\right)\left(1-\epsilon_{\mathrm{D}-\mathrm{C}^{\prime}}\right)^{2},
$$

where

$$
\begin{gathered}
\epsilon_{\mathrm{D}-\mathrm{C}}=1-\exp \left[-\left(P_{\max } X\right) \operatorname{erfc}(a) \exp \left(a^{2}\right)\right], \\
\epsilon_{\mathrm{D}-\mathrm{C}^{\prime}}=1-\exp \left[-\left(P_{\max } X^{\prime}\right) \operatorname{erfc}\left(a^{\prime}\right) \exp \left(a^{\prime 2}\right)\right],
\end{gathered}
$$

and $a^{\prime}$ represents the line-shape parameter for the gases at the temperature $T^{\prime}$.

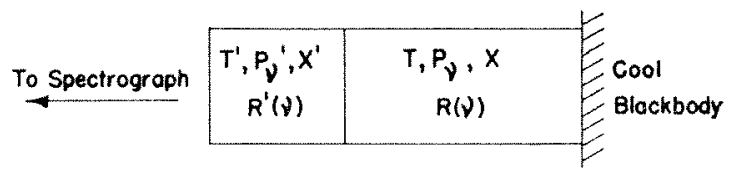

Fic. 9. Schematic arrangement for emission experiment from two adjacent isothermal regions.

The ratio $I / I^{\prime}$ has been calculated for $a^{\prime}=a$ ranging from 0 to $10, \epsilon(K=1)=0.7, \epsilon^{\prime}(K=1)=0.1$ and 0.5 , for the $P_{1}$ branch, $(0,0)$ band, and ${ }^{2} \mathrm{\Sigma} \rightarrow{ }^{2} I I$ transitions of $\mathrm{OH}$. Results are plotted in Figs. 7 and 8. Reference to Figs. 7 and 8 shows that two-path experiments will yield results which are sensitive functions of the lineshape parameter $a$ with distortion of experimental data by self-absorption diminishing as the numerical value of $a$ is increased, under otherwise comparable experimental conditions.

\section{PEAK ABSORPTION EXPERIMENTS FOR TWO ADJACENT ISOTHERMAL REGIONS (SPECTRAL LINES WITH COMBINED DOPPLER- AND COLLISION-BROADENING)}

Calculations in this section correspond to the experimental arrangement illustrated in Fig. 6 with $P_{\max }$ replaced by $P\left(v_{l t}\right)$. Equation (4) now becomes

$$
I^{*} / R\left(T_{\mathrm{s}}\right) \simeq\left(1-\epsilon_{\mathrm{D}-\mathrm{C}}\right)\left(1-\epsilon_{\mathrm{D}-\mathrm{C}^{\prime}}\right)^{2} .
$$

Comparison of Eqs. (3a) and (4a) shows that $I^{*} / R\left(T_{s}\right)$ $=I / I^{\prime}-1$ is obtained simply by reinterpreting the ordinates of Figs. 7 and 8 . Hence the same conclusions apply to absorption experiments with discrete sources as 
to measurements of peak intensities for two-path experiments.

\section{TOTAL INTENSITIES FOR EMISSION OF RADIATION FROM FLAMES CONSISTING OF TWO ADJACENT ISOTHERMAL REGIONS (SPECTRAL LINES WITH DOPPLER CONTOUR)}

The experimental arrangement is sketched in Fig. 9. The total observable intensity 1 for the line with center at $\nu_{l u}$ is

$$
\begin{aligned}
A \equiv \int_{-\infty}^{\infty} I_{v} d v \simeq R\left(\nu_{l u}\right) \\
\quad \times \int_{-\infty}^{\infty}\left[1-\exp \left(-P_{v} X\right)\right]\left[\exp \left(-P_{v}^{\prime} X^{\prime}\right)\right] d v,
\end{aligned}
$$

where the intensity of radiation emitted from the cool

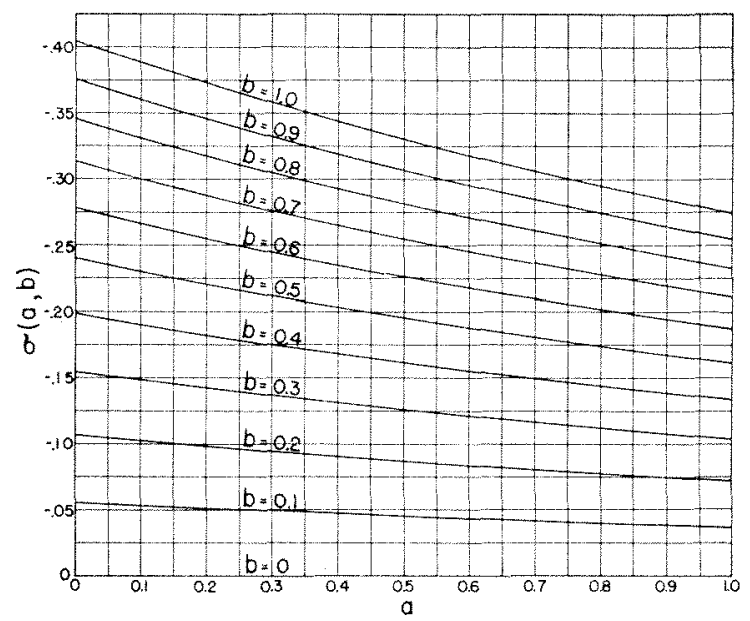

Fic. 10. The double sum $\sigma(a, b)$ for $c=2$ ats a function of $a$ and $b$.

gas layer has been neglected. But, for spectral lines with Doppler contour, $P_{\nu}=a \exp \left[-\beta\left(\nu-\nu_{l u}\right)^{2}\right], P_{v}^{\prime}$ $=b \exp \left[-\beta^{\prime}\left(\nu-\nu_{l u}\right)^{2}\right]$, where $a=P_{\max } X, b=P_{\max } X^{\prime}$, $\beta=m c^{2} 2 k T \nu_{l u^{2}}{ }^{2}, \beta^{\prime}=m c^{2} 2 k T^{\prime} \nu_{l u}{ }^{2}$. Replacing $P_{y}$ and $P_{v}^{\prime}$ and making the substitutions, $x^{2}=\beta\left(\nu-\nu_{l}\right)^{2}, d \nu$ $=\beta^{-(1 / 2)} d x, \beta^{\prime}\left(\nu-\nu_{l u}\right)^{2}=\left(\beta^{\prime} / \beta\right) x^{2} \equiv c x^{2}$, and expanding the exponentials, it follows that

$$
\begin{aligned}
A / R\left(\nu_{n}\right)= & P_{\max } X(\pi / \beta)^{(1 / 2)} \sum_{n=0}^{\infty}\left[(-1)^{n}\left(P_{\max } X\right)^{n}\right] \\
& {\left[(n+1) !(n+1)^{(1 / 2)}\right]+P_{\max } X(\pi / \beta)^{(1 / 2)} } \\
& \times \sum_{n=0=0}^{\infty} \sum_{n=1}^{\infty}(-1)^{n+m}\left(P_{\operatorname{mix}} X\right)^{n}\left(P_{\max } X^{\prime}\right)^{m} \\
& \times\left[(n+1) ! m !(n+1+m)^{(1 / 2}\right]^{-1}
\end{aligned}
$$

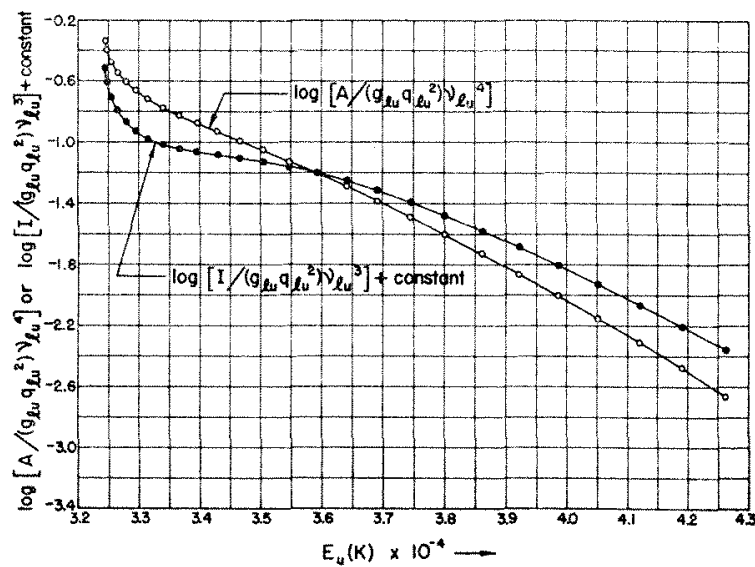

Fig. 11. Comparison of conventional plots for the determination of rotational temperatures of OH for measurements of total emitted intensities $A$ and of peak emitted intensities $l$. Two adjacent isothermal regions at temperatures of 3000 and $1500^{\circ} \mathrm{K}$. respectively; spectral lines with Doppter contour; $\epsilon=0.5, \epsilon^{\prime}=0.3$.

But $S_{\operatorname{lu}} X=P_{\max } X(\pi / \beta)^{\frac{1}{2}}$, whence

$$
\begin{aligned}
A / R\left(\nu_{l u}\right)= & S_{l u} X\left\{\sum_{n=0}^{\infty}(-1)^{n}\left(P_{\max } X\right)^{n}\right. \\
& \times\left[(n+1) !(n+1)^{\frac{1}{2}}\right]^{-1} \\
& +\sum_{n=0}^{\infty} \sum_{m=1}^{\infty}(-1)^{n+m}\left(P_{\max } X\right)^{n}\left(P_{\max } X^{\prime}\right)^{m} \\
& \left.\times\left[(n+1) ! m !(n+1+m c)^{(1 / 2)}\right]^{-1}\right\}
\end{aligned}
$$

The value of the first sum appearing on the righthand side of Eq. (9) has been determined by Ladenburg as a function of $P_{\min x} X{ }^{7}$ It therefore remains to calculate the value of the double sum, $\sigma(a, b, c)$, as a function of $a=P_{\max } X$ and $b=P_{\max }{ }^{\prime} X^{\prime}$. For small values of $a$ and $b$ the double sum given in Eq. (9) can be evaluated conveniently by direct summation of the rapidly convergent series. The results of the calculations for $c=T / T^{\prime}=2$ are plotted in Fig. 10 where the ordinate, $\sigma(a, b)$, represents the double sum appearing on the right-hand side of Eq. (9) for $c=2$.

The ratio $A / R\left(\nu_{l u}\right)$ has been computed from Eq. (9) as a function of $a$ and $b$ for $\epsilon=1-\exp (-a)=0.5$ and $\epsilon^{\prime}=1-\exp (-b)=0.3$ for the $P_{1}$ branch, $(0,0)$ band, $2 \Sigma \rightarrow \rightarrow^{2}$ Iransitions of $\mathrm{OH}$. The results of these calculations are contrasted in Fig. 11 with the corresponding data applying to peak intensities. ${ }^{2}$ Reference to Fig. 11 shows that, for spectral lines with Doppler contour, distortion of data for measurements of total intensity is smaller than the distortion calculated for peak intensities. For rotational temperatures derived from emission experiments on isothermal regions, very similar results had been obtained previously for measurements of total and of peak emission intensities."

\footnotetext{
${ }^{7}$ R. Ladenburg, Z. Physik 65, $200(1930)$
} 